\title{
EVOLUÇÃOTEMPORAL DA RESISTÊNCIA IN VTTRO DO PLASMODIUM FALCIPARUM ÀS DROGAS ANTIMALÁRICAS EM DUAS ÁREAS DAAMAZÔNIA BRASILEIRA COM DISTINTAS CARACTERÍSTICAS SÓCIO-ECONÔMICAS E GEOGRÁFICAS
}

\author{
Álvaro Augusto Couto, Vanja Suely Calvosa, Marco Antonio Santos e \\ José Maria de Souza
}

\begin{abstract}
Avaliou-se a evolução temporal da resistência in vitto do Plasmodium falciparum às drogas cloroquina, amodiaquina, quinino e mefloquina em duas áreas com distintas características sócio-econômicas e geográficas: Lourenço, no Estado do Amapá e Paragominas no Estado do Pará. A primeira caracteriza-se por ser uma área de garimpos a céu aberto e a segunda uma área de colonização, pecuária e extrativismo de madeiras. O estudo revela alta prevalência de resistência à cloroquina nas duas äreas $(79,8 \%$ em Lourenço e $68,4 \%$ em Paragominas), enquanto que para amodiaquina e quinino observamos uma certa flutuação nas respostas para essas drogas, dependendo do periodo em que foi avaliada. Já para mefloquina, não foram observadas cepas resistentes, mas uma perda da sensibilidade ao longo do periodo estudado.
\end{abstract}

Palavras-chaves: Malária. Plasmodium falciparum. Resistência in vitro.

O principio básico nos programas contra a malária, no Brasil, visa ao controle da doença, com objetivos específicos de reduzir sua incidência nas áreas de elevada transmissão, e, interrompê-la nas regiôes de incidência baixa, além de evitar que ocorra a reintrodução onde a malária não mais existe. No entanto, têm sido frequientes os insucessos, parciais ou totais, das medidas de controle da malária, particularmente, em algumas áreas da Região Amazônica Brasileira. Nos últimos anos constata-se a expansão territorial da malária, o ressurgimento da transmissão urbana, evidenciada por exemplo, em Manaus, AM, a partir de 1986 e, mais recentemente, em Belém, PA verifica-se o aumento exagerado da autoctonia da doença.

Fatores geográficos e sócio-econômicos estão fortemente associados a esse processo, no entanto, características peculiares aos parasitas e vetores são determinantes de obstáculos para o estabelecimento de um

Serviço de Ecologia Humana e Meio Ambiente e Serviço de parasitologia, Instituto Evandro Chagas, Fundação Nacional de Saúde (FNS), Belém, PA.

Apoio Financeiro: IEC-FINEP - UNDP/World Bank/ WHO/TDR, Projeto (870284).

Endereço para correspondência: Dr. Alvaro A. Couto. Av. Almirante Barroso 492, Marco, 66090-000 Belém, PA.

Recebido para publicação em 22/12/94. efetivo controle da doença. Assim, os parasitas têm demonstrado um elevado grau de resistência cruzada às drogas empregadas e os vetores parecem mostrar uma crescente adaptabilidade às modificações ambientais resultantes da implantação dos projetos de colonização, agropecuários e de mineração.

A resistência do Plasmodium falciparum às drogas antimaláricas constitui um dos maiores obstáculos para um efetivo controle da doença?. No Brasil esta resistência foi demonstrada, pela primeira vez, em 1910 para o quinino" ${ }^{11}$ Em 1954, Brito e Pinheiro ${ }^{1}$ reportaram, na Amazônia Brasileira, a ineficácia da cloroquina em alguns casos de malária causada pelo $P$. falciparum. Novamente foi registrada a ocorrência de resistência à cloroquina em 196120 21. Uma ampla e rápida disseminação da resistência, detectada in vivo e in vitro, tem sido documantada nos últimos 10 anos em diversas áreas da Amazônia Brasileiraa 3182225.

A distribuição da malária na Amazônia Brasileira não é homogênea. Localizada em determinadas áreas com características peculiares, notadamente áreas de colonização e prospeç̧ão de ouro', algumas possuem maior comprometimento pelo elevado nivel de transmissão e resistência dos parasitas aos antimaláricos de uso rotineiro no tratamento 
Couto AA, Calvosa VS, Santos MA, Souza JM. Evolução temporal da resistência in vivo do Plasmodium falciparum às drogas antimaláricas em duas áreas da Amazônia Brasileira com distintas caracteristicas sócio-econômicas e geográficas. Revista da Sociedade Brasileira de Medicina Tropical 28:357-365, out-dez, 1995.

da doença ${ }^{32}$. Considerando as peculiaridades dessas áreas é possível que ocorram variações no padrão, evolução e mecanismo de disseminação da resistência do P. falciparum. A variabilidade das características das cepas brasileiras foram bem documentadas no período de 1983 à $1987^{4561517}$.

Objetivamos avaliar a evolução temporal da resistência in vitro e possíveis influências de características sócio-econômicas e comportamentais da população, como fatores determinantes de diferenças no padrão de respostas às drogas. Para isto, avaliamos, no período de 1983 a 1991, o perfil da resistência in vitro para as drogas cloroquina, amodiaquina, quinino e mefloquina em duas áreas distintas da Região Amazônica Brasileira: Lourenço, AP com exclusiva atividade de prospecção de ouro a céu aberto, e, Paragominas, PA, área de colonização, agropecuária e ampla atividade de extrativismo de madeira

\section{MATERIAL E MÉTODOS}

O estudo foi desenvolvido em duas áreas que apresentam diferentes características geográficas e sócio-econômicas, aqui denominadas área 1 (Lourenço, AP) e área 2 (Paragominas, PA).

Área 1. O distrito do Lourenço possui as seguintes coordenadas geográficas: $2^{\circ} 18^{\prime} 17^{\prime \prime}$ de latitude (S) e 5138'09" de longitude (W); situase ao Norte do Estado do Amapá e dista, em Iinha reta, $240 \mathrm{~km}$ da capital Macapá, com uma área de $280 \mathrm{~km}^{2}$ pertencendo ao Município de Calçoene, Estado do Amapá. Caracteriza-se pela intensa e exclusiva atividade de prospecção de ouro a céu aberto, com uma população muito instável face à intensa migração, peculiar em áreas de garimpagem na Amazônia. No período de 1985 a 1988 registrava-se uma população entre 6.000 a 10.000 habitantes; nos últimos dois anos do estudo foi reduzida para 2.000 habitantes em funçâo da escassez do ouro. Os habitantes vivem de forma precária, em habitações sem paredes, o que facilita o contato com o vetor e diminui a possibilidade de sua proteção com inseticida residual. Esse é portanto, um dos fatores epidemiológicos da mais alta importância, possivelmente, o principal responsável pelo alto nivel de transmissão da malária na região. Segundo Sawyer ${ }^{19}$, a região se enquadra na classificação "área rural extrativista garimpo aberto - tipo 2".

O clima é tropical chuvoso, possui precipitações anuais em torno de $3.069 \mathrm{~mm}$ com temperatura média mensal de $25,6^{\circ} \mathrm{C}$, com média anual de $24^{\circ} \mathrm{C}$, com máxima e mínima de $30^{\circ} \mathrm{C}$ e $18^{\circ} \mathrm{C}$, respectivamente. A umidade relativa do ar é superior a $75 \%$ com altitude de $305 \mathrm{~m}$ acima do nível do mar.

Área 2. O Município de Paragominas possui as seguintes coordenadas geográficas: $2^{\circ} 54^{\prime} 43^{\prime \prime}$ de latitude (S) e 4721'12" de longitude (W); situa-se à leste do Estado do Pará e dista em linha reta $205 \mathrm{~km}$ da capital Belém, com uma área de $27.168 \mathrm{~km}^{2}$. Paragominas, município do Estado do Pará, devido a sua localização geográfica, interiorizado, possui características próprias em suas atividades econômicas. No setor primário, destaca-se a exploração da pecuária, produção agrícola e o extrativismo da madeira. Possui amplo parque industrial formado por indústrias de beneficiamento de madeiras (serrarias) e usinas de beneficiamento de arroz. Pelos dados do IBGE, censo de 1991, o município apresentou uma população de 92.173 habitantes. A população do município se dispersa no seu vasto território dando origem à formação de pequenas vilas e colônias, vivendo em casas de alvenaria, na cidade, e no campo em habitações de madeira. Segundo Sawyer ${ }^{19}$, o Município de Paragominas se enquadra na classificação de "área rural agricultural agropecuária (fazendas) Tipo 7".

O clima é tropical chuvoso com precipitações anuais acima de $750 \mathrm{~mm}$, com temperatura média mensal de $18^{\circ} \mathrm{C}$. Apresenta uma estação relativamente seca, compensada por precipitações elevadas durante o período restante. A temperatura média anual é de $25^{\circ} \mathrm{C}$, com máxima e mínima de $31^{\circ} \mathrm{C}$ e $22^{\circ} \mathrm{C}$, respectivamente. A umidade relativa do ar é superior a $80 \%$ e o municipio situa-se a $84 \mathrm{~m}$ acima do nível do mar.

População estudada. Os indivíduos doadores de sangue para o presente estudo foram de ambos os sexos, com idades variando de 13 a 48 anos, residentes na Vila do Lourenço e adjacências (garimpos) ou no Município de Paragominas nas diversas colônias ou fazendas.

Durante o período de estudo foram triados 569 pacientes da área 1 e 621 da área 2, dos quais foram coletados $10 \mathrm{ml}$ de sangue para obtenção do material necessário para a 
Couto AA, Calvosa VS, Santos MA, Souza JM. Evolução temporal da resistência in vivo do Plasmodium falciparum ãs drogas antimaläricas em duas áreas da Amazônia Brasileira com distintas características sócio-econômicas e geográficas. Revista da Sociedade Brasileira de Medicina Tropical 28:357-365, out-dez, 1995.

realização dos microtestes, criopreservação das cepas e soro para estudos complementares.

Para avaliação da resistência in vitro foram selecionados àqueles portadores exclusivamente de malária causada pelo $P$. falciparum com parasitemia superior a 500 parasitas por $\mu^{1} \mathrm{l}$ de sangue e não apresentando quadro de malária grave. Quando a parasitemia apresentava-se igual ou superior a $1 \%$ ajustava-se a amostra de sangue parasitado em torno de $0,5 \%$

Todos os participantes declararam o não uso de drogas antimaláricas nas últimas quatro semanas, além de darem consentimento oral para sua inclusão no estudo. Após a coleta da amostra sanguínea todos foram tratados e acompanhados até a completa cura.

Testes in vitro de sensibilidade as drogas. Os testes in vitro foram realizados com base no procedimento de Rieckmann ${ }^{14}$, com as modificações introduzidas pela $\mathrm{OMS}^{26}{ }^{27}$. No período de 1983 a $1986^{18}$ utilizou-se o sistema de diluições no momento da realização dos testes, partindo-se de uma solução "stock" fornecida pela OMS. No período de 1987 a $1991^{2}$, foram utilizadas placas prédoseadas, também, fornecidas pela OMS.
A interpretação dos resultados foi realizada segundo as instruções da OMS 122728 .

\section{RESULTADOS}

Foram realizados 85 testes para avaliar a resistência do P. falciparum em Lourenço, AP (Área 1), dos quais, tiveram boas condições para interpretação; 84 para cloroquina, 82 para amodiaquina, 83 para quinino e 85 para mefloquina. As amostras analisadas em Paragominas, PA (Área 2) foram 76, das quais 76 estavam em boas condições de análise para cloroquina, 54 para amodiaquina, 57 para quinino e 76 para mefloquina.

Os niveis de resistência nas áreas em estudo foram: Área 1, com 67 amostras correspondentes a $79,8 \%$ de cepas resistentes à cloroquina e 22 amostras correspondendo a $26,8 \%$ de resistência à amodiaquina, porém não foram evidenciadas amostras resistentes ao quinino e à mefloquina; Área 2, com 52 amostras equivalendo a $68,4 \%$ de cepas resistentes à cloroquina, 26 amostras $(48,1 \%)$ à amodiaquina e 8 amostras $(14,0 \%)$ ao quinino, enquanto que para mefloquina não foram registradas cepas resistentes (Tabela 1 ).

Tabela 1 - Resistência do Plasmodium falciparum em duas diferentes áreas da Região Amazônica às drogas cloroquina, amodiaquina, quinino e mefloquina no período de 1983 a 1991.

\begin{tabular}{|c|c|c|c|c|c|c|c|c|c|c|c|c|}
\hline \multirow{3}{*}{$\begin{array}{l}\text { Areas de } \\
\text { estudo }\end{array}$} & \multicolumn{12}{|c|}{ Drogas } \\
\hline & \multicolumn{3}{|c|}{ cloroquina } & \multicolumn{3}{|c|}{ amodiaquina } & \multicolumn{3}{|c|}{ quinino } & \multicolumn{3}{|c|}{ mefloquina } \\
\hline & $\overline{n^{2}}$ & $r$ & $\%$ & $n^{9}$ & $T$ & $\%$ & $\mathrm{n}^{2}$ & $r$ & $\%$ & $\mathrm{n}^{\varrho}$ & $\mathrm{r}$ & $\%$ \\
\hline \multicolumn{13}{|c|}{$\overline{\text { Lourenço, } \mathrm{AP}}$} \\
\hline Área 1 & 84 & 67 & 80 & 82 & 22 & 27 & 83 & 0 & 0 & 85 & 0 & 0 \\
\hline \multicolumn{13}{|c|}{ Paragominas, PA } \\
\hline Área 2 & 76 & 52 & 68 & 54 & 26 & 48 & 57 & 8 & 14 & 76 & 0 & 0 \\
\hline
\end{tabular}

Nas amostras da Área 1 não se observou resistência ao quinino, mas evidenciou-se que $11(13,6 \%)$ cepas só foram completamente inibidas na concentração limite da sensibilidad̦e/resistência, ou seja 128pmol.

A análise tempòral do perfil da resistência nas amostras de ambas as áreas no periodo de 1983 à 1991, revela que existe uma aparente semelhança no padrão de resposta do $P$. falciparum às drogas cloroquina e quinino, e, amodiaquina e mefloquina, como se pode verificar através das Figuras $1 \mathrm{a}, 1 \mathrm{~b}$ e $2 \mathrm{a}, 2 \mathrm{~b}$.

Existem certas peculiaridades, em cada uma das duas áreas estudadas com relação à evolução da resistência às drogas cloroquina, amodiaquina, quinino e mefloquina.
A resistência à cloroquina na Área 1 aparentemente evoluiu de forma mais rápida do que na Área 2.

Para a amodiaquina, apesar de ser uma 4aminoquinoleína como a cloroquina, o perfil de comportamento da população de parasitas foi diferente nas áreas e reflete, possivelmente, uma maior ou menor pressão da droga, nas respectivas áreas em estudo, como será discutido.

Com relação ao quinino, em Lourenço não se observou resistência, mas houve uma evidente redução da sensibilidade à droga ao longo dos anos. Para mefloquina existe uma diminuição mais precoce da sensibilidade em Lourenço, AP, em relação ao padrão da Área 2. 
Couto AA, Calvosa VS, Santos MA, Souza JM. Evolução temporal da resistência in vivo do Plasmodium falciparum às drogas antimaláricas em duas áreas da Amazônia Brasileira com distintas características sócio-econômicas e geográficas. Revista da Sociedade Brasileira de Medicina Tropical 28:357-365, out-dez, 1995.

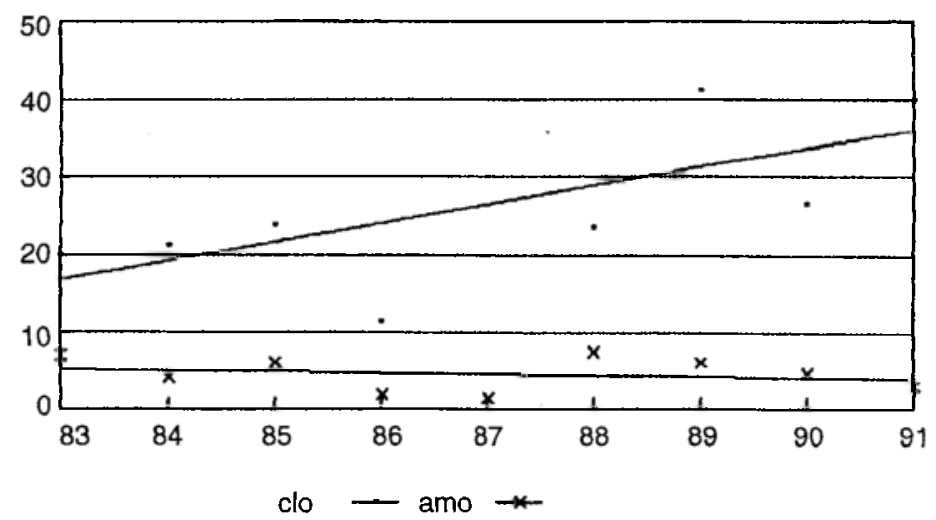

Figura 1a - Evolução da resistência do P. falciparum à cloroquina $e$ amodiaquina Lourenç, AP, período 1983 a 1991.

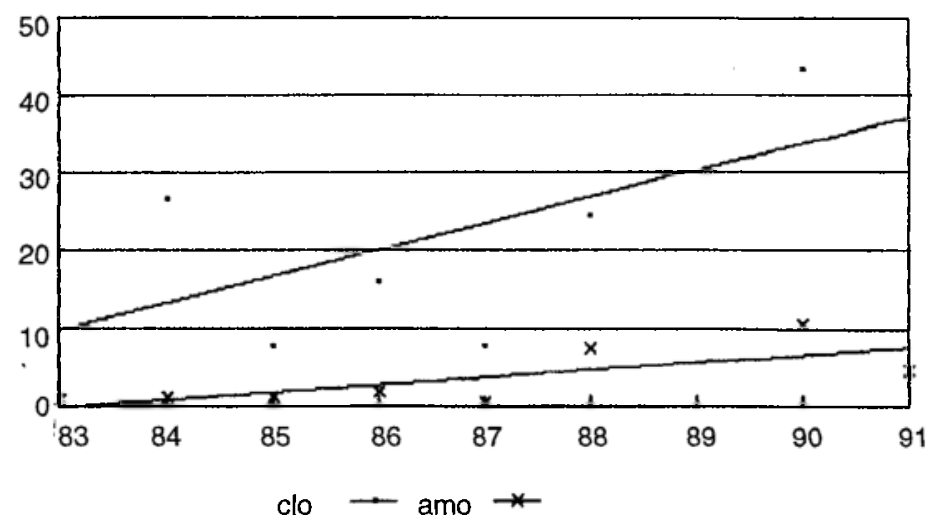

Figura $1 b$ - Evolução da resistência do P. falciparum à cloroquina e amodiaquina Paragominas, AP, periodo 1983 a 1991.

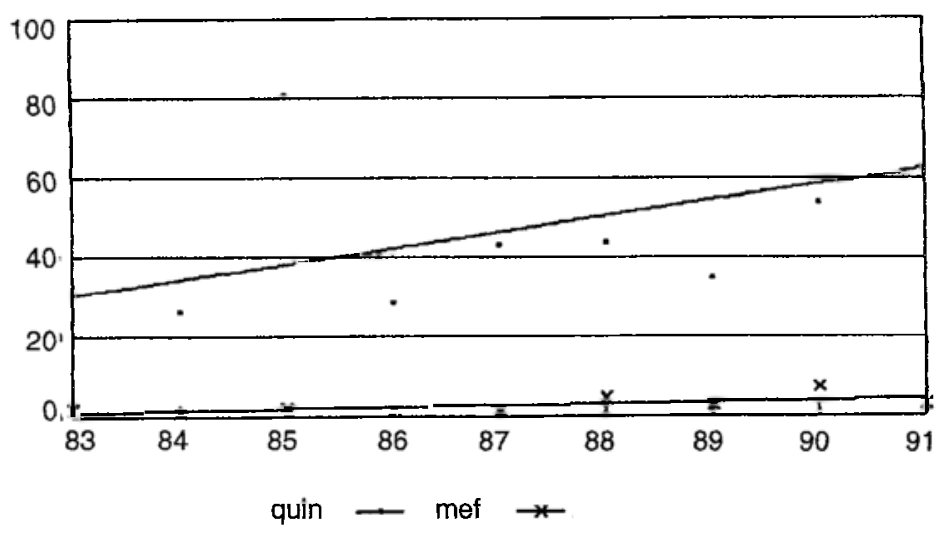

Figura 2a - Evolução da resistência do P. falciparum ao quinino e à mefloquina, Lourenço, AP, periodo 1983 a 1991. 
Couto AA, Calvosa VS, Santos MA, Souza JM. Evolução temporal da resistência in vivo do Plasmodium falciparum às drogas antimaläricas em duas âreas da Amazônia Brasileira com distintas características sócio-econômicas e geográficas. Revista da Sociedade Brasileira de Medicina Tropical 28:357-365, out-dez, 1995.

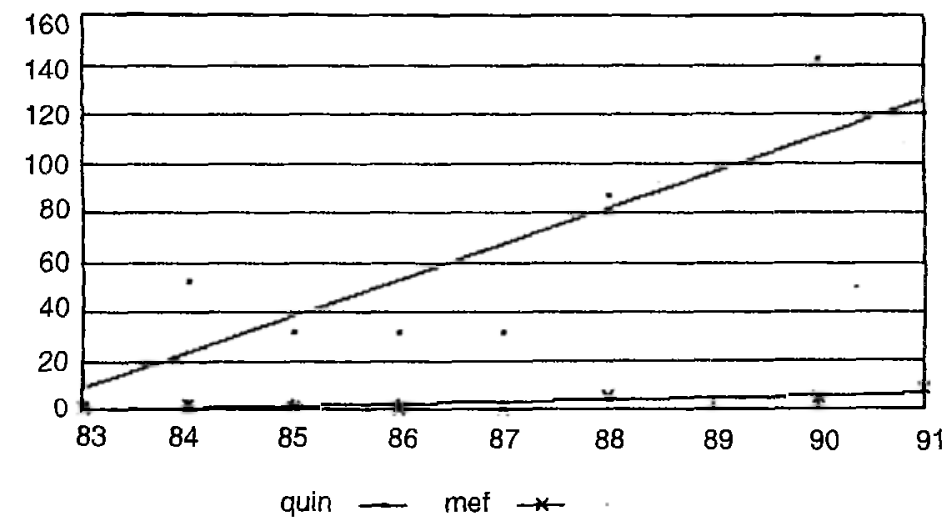

Figura $2 b$ - Evolução da resistência do P. falciparum ao quinino e à mefloquina, Paragominas, $A P$, periodo 1983 a 1991.

Nossos resultados são similares àqueles observados em outras localidades da Região Amazônica Brasileira, quer por estudos realizados por nosso grupo, quer por aqueles realizados por outros pesquisadores, conforme consta da Tabela 2.

Tabela 2 - Percentagem de cepas de Plasmodium falcjparum resistentes à diferentes drogas na Região Amazônica Brasileira no periodo de 1983 à 1993 e referências bibliográficas.

Areas $\quad$ Drogas (\%) Referencias

\begin{tabular}{|c|c|c|c|c|c|}
\hline & clo & amo & qui & mef & \\
\hline $\begin{array}{l}\text { Pará, Amapá, } \\
\text { Rondônia }\end{array}$ & 68 & 9 & 0 & 0 & 25 \\
\hline Pará, Amapá, & & & & & \\
\hline Rondônia, Maranhão & 83 & 51 & 56 & 2 & 18 \\
\hline $\begin{array}{l}\text { Rondônia, Goiás, } \\
\text { Mato Grosso, Pará, }\end{array}$ & & & & & \\
\hline Acre & 100 & $\mathrm{nr}$ & 2 & 31 & 6 \\
\hline Acre & 84 & 73 & 3 & 0 & 8 \\
\hline Amapá & 81 & 27 & 0 & 0 & 2 \\
\hline Pará. & 71 & 26 & 8 & 0 & 3 \\
\hline
\end{tabular}

clo: cloroquina; amo: amodiaquina; qui: quinino; mef: mefloquina

\section{DISCUSSÃO}

Diversos fatores estão associados à emergência e disseminação da resistência dos parasitos da malária às drogas, sendo provavelmente um dos mais importantes à pressão de drogas ${ }^{29}$. Nossos resultados evidenciam esse fenômeno em duas áreas da Amazônia com todas as drogas estudadas. No presente trabalho, a resposta in vitro dos parasitas às quatro drogas estudadas, duas $4-$ aminoquinoleínas e dois quinolinometanóis, é documentada ao longo de 9 anos e mostra características peculiares para cada uma das drogas. Essas parecem refletir critérios utilizados pelos órgãos governamentais responsáveis pelo controle da malária no Brasil, relacionados com o uso dos antimaláricos e com as facilidades existentes na obtenção dos mesmos. No entanto, não parece haver relação entre o perfil geográfico e/ou sócio-econômico das duas áreas avaliadas e a resistência aos antimaláricos.

A cloroquina era a droga mais utilizada em ambas as áreas muito antes de 1983 , portanto houve uma constante pressão dessa droga sobre as populaçôes de parasitas. Como conseqüência a maioria das amostras testadas $(67 / 87,80 \%)$ está acima do limiar da sensibilidade, na Área 1, enquanto que na Área $2,52 / 76(68 \%)$ foram resistentes. A sensibilidade à cloroquina foi semelhante nas duas localidades nas quais não há diferenças geográficas marcantes, mas onde os perfis sócio-econômicos são distintos. Os critérios do Ministério da Saúde foram iguais com relação ao uso de droga em grande escala. Portanto, apesar das diferenças sócio-econômicas e, das dificuldades logísticas, as áreas não receberam diretrizes diferentes do governo em relação às metodologias de controle da malária.

A utilização da amodiaquina e os níveis de resistência foram diferentes nas duas áreas. Na Área 1, seu uso não foi uma prática consistente no período em estudo, por não haver disponibilidade da droga ou porque nela não 
Couto AA, Calvosa VS, Santos MA, Souza JM. Evolução temporal da resistência in vivo do Plasmodium falciparum d̀s drogas antimaläricas em duas áreas da Amazônia Brasileira com distintas características sócio-econômicas e geográficas. Revista da Sociedade Brasileira de Medicina Tropical 28:357-365, out-dez, 1995.

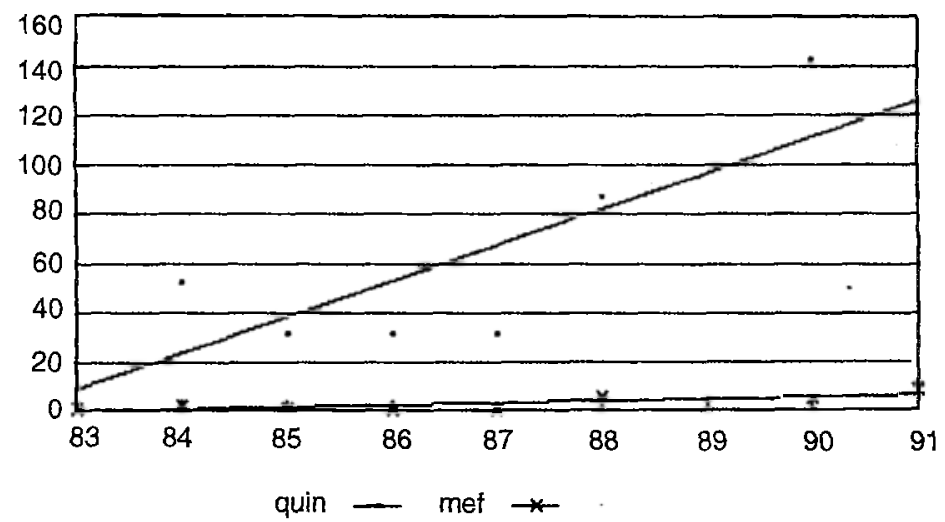

Figura $2 b$ - Evolução da resistência do P. falciparum ao quinino e à mefloquina, Paragominas, AP, periodo 1983 a 1991.

Nossos resultados são similares àqueles observados em outras localidades da Região Amazônica Brasileira, quer por estudos realizados por nosso grupo, quer por aqueles realizados por outros pesquisadores, conforme consta da Tabela 2.

Tabela 2 - Percentagem de cepas de Plasmodium falciparum resistentes à diferentes drogas na Região Amazônica Brasileina $n$ periodo de 1983 à 1993 e referencias bibliográficas.

\begin{tabular}{|c|c|c|c|c|c|}
\hline Areas & & Drog & $(\%)$ & & Referências \\
\hline & clo & amo & qui & mef & \\
\hline
\end{tabular}

\begin{tabular}{lrrrrr}
\hline $\begin{array}{l}\text { Pará, Amapá, } \\
\quad \begin{array}{l}\text { Rondônia } \\
\text { Pará, Amapá, }\end{array} \\
\quad \text { Rondônia, Maranhào }\end{array}$ & 83 & 51 & 56 & 2 & 18 \\
$\begin{array}{l}\text { Rondônia, Goiás, } \\
\quad \text { Mato Grosso, Pará. }\end{array}$ & & & & & \\
$\quad$ Acre & 100 & nr & 2 & 31 & 6 \\
Acre & 84 & 73 & 3 & 0 & 8 \\
Amapá & 81 & 27 & 0 & 0 & 2 \\
Pará & 71 & 26 & 8 & 0 & 3 \\
\hline
\end{tabular}

clo: cloroquina; amo: amodiaquina; qui: quinino; mef: mefloquina

\section{DISCUSSÃO}

Diversos fatores estão associados à emergência e disseminação da resistência dos parasitos da malária às drogas, sendo provavelmente um dos mais importantes à pressāo de drogas ${ }^{29}$. Nossos resultados evidenciam esse fenômeno em duas áreas da Amazônia com todas as drogas estudadas. No presente trabalho, a resposta in vitro dos parasitas às quatro drogas estudadas, duas 4- aminoquinoleínas e dois quinolinometanóis, é documentada ao longo de 9 anos e mostra características peculiares para cada uma das drogas. Essas parecem refletir critérios utilizados pelos órgãos governamentais responsáveis pelo controle da malária no Brasil, relacionados com o uso dos antimaláricos e com as facilidades existentes na obtenção dos mesmos. No entanto, não parece haver relação entre o perfil geográfico e/ou sócio-econômico das duas áreas avaliadas e a resistência aos antimaláricos.

A cloroquina era a droga mais utilizada em ambas as áreas muito antes de 1983, portanto houve uma constante pressão dessa droga sobre as populações de parasitas. Como conseqüencia a maioria das amostras testadas (67/87, 80\%) está acima do limiar da sensibilidade, na Área 1, enquanto que na Área $2,52 / 76(68 \%)$ foram resistentes. A sensibilidade à cloroquina foi semelhante nas duas localidades nas quais não há diferenças geográficas marcantes, mas onde os perfis sócio-econômicos são distintos. Os critétios do Ministério da Saúde foram iguais com relação ao uso de droga em grande escala. Portanto, apesar das diferenças sócio-econômicas $e$, das dificuldades logísticas, as áreas não receberam diretrizes diferentes do governo em relação às metodologias de controle da malária.

A utilização da amodiaquina e os níveis de resistência foram diferentes nas duas áreas. $\mathrm{Na}$ Área 1, seu uso não foi uma prática consistente no período em estudo, por não haver disponibilidade da droga ou porque nela não 
Couto AA, Calvosa VS, Santos MA, Souza JM. Evolução temporal da resistência in vivo do Plasmodium falciparum às drogas antimalāricas em duas âreas da Amazônia Brasileira com distintas caracteristicas sócio-econômicas e geográficas. Revista da Sociedade Brasileira de Medicina Tropical 28:357-365, out-dez. 1995.

se confiava como alternativa para substituir a cloroquina. Apesar da semelhança química, entre as duas drogas, que poderia levar a uma resistência cruzada com a cloroquina, manteve-se uma estabilidade nos níveis de resistência e até mesmo uma tendência à sua redução, provavelmente devido à pouca pressão da droga sobre os parasitas.

$\mathrm{Na}$ Área 2, apesar do número reduzido de amostras estudadas (aproximadamente $2 / 3$ do número de amostras analisadas na Área 1), a amodiaquina foi gradativamente menos ativa nos testes. A princípio, essa droga era mais utilizada na Área 2 e mostrava maior atividade sobre os parasitas.

A partir de 1988, essa atividade diminui observando-se ao final do estudo uma sensibilidade correspondente a 10 ou $20 \%$ do total de amostras analisadas. Esta mudança coincide com o maior uso da amodiaquina em Paragominas, onde antes se usava exclusivamente a cloroquina ou a cloroquina associada com à primaquina (cloroprima), nas fases de maior transmissão da doença. Essa observação mais uma vez caracteriza a pressão da droga sobre os parasitas como fator de importância na emergência da resistência mostrada.

O quinino, como a cloroquina, apresenta um padrão de uso semelhante nas duas áreas estudadas. Contudo, ao contrário da cloroquina, a sensibilidade ao quinino é evidente, praticamente em todas as cepas estudadas in vitro apesar de se verificar, em ambas as áreas, uma progressiva redução da sensibilidade. Possivelmente, há dois fatores atuando, ou seja, a pressão da droga, em função do descrédito das 4-aminoquinoleínas, e o esquema terapêutico em subdoses adotados pelos órgãos governamentais e, naturalmente, também utilizado pela grande maioria das clínicas da rede privada, correspondente a uma quantidade da substância ativa pouco ou muito abaixo da dose preconizada, dependendo do peso do paciente, ou seja, mantinham a pressão da droga com dosagem insuficiente.

Para mefloquina, apesar de não se registrarem cepas resistentes, verificou-se, uma tendência à perda de sensibilidade em ambas as áreas. Essa começou mais precocemente na Área 1, já em 1985, e, alguns anos depois na Área 2. Paralelamente, enquanto a população da Área 1 teve acesso fácil à droga a partir de
1985, através da entrada clandestina da mefloquina, via Guiana Francesa, só em 1987 a população da Área 2 recebeu a mefloquina pelo programa do Ministério da Saúde/SUCAM. A ocorrência de pressão da droga em diferentes épocas nas duas áreas provavelmente provocou a seleção de parasitas menos sensíveis em épocas diferentes nas duas áreas.

Além da pressão da droga, sua inadequada concentração pode contribuir para a seleção de parasitas resistentes. Em áreas de garimpo a utilização de drogas antimaláricas em dosagens subcurativas é mais freqüente do que em outras áreas, fato esse comum a todas as áreas malarígenas onde a transmissão é elevada.

Segundo constatamos, a automedicação foi aparentemente mais freqüente na Área 1, quase sempre incorreta e envolvendo várias drogas, inclusive a mefloquina e derivados da artemisinina (observação pessoal). Esta prática leva freqüentemente ao insucesso terapêutico, favorecendo, portanto, o surgimento da resistência, uma vez que na população de parasitas remanescentes são selecionados parasitas resistentes ${ }^{316172326}$.

Possivelmente, a pressão de drogas, doses subcurativas e automedicação sejam os principais fatores responsáveis pela perda da eficácia dos antimaláricos, mesmo da mefloquina. Nossos resultados mostram uma perda de sensibilidade mais precoce na Área 1, em relação à Área 2. Aí, a droga foi introduzida a partir de 1987, após o Plano Impacto de Controle da Malária, elaborado pelo MS/SUCAM ${ }^{10}$.

Nossos resultados evidenciam a evolução da resistência em duas áreas distintas da Amazônia Brasileira, escolhidas por representarem os ambientes mais problemáticos ao controle da malária. A população de parasitas circulantes nestas áreas exibiu nível de resistência à cloroquina na ordem de 79,8\% e 68,4\% em Lourenço, AP e Paragominas, $\mathrm{PA}$, respectivamente, similar ao que tem sido descrito em outras áreas da Região Amazônica brasileira, por nosso grupo ${ }^{2318}$ e por outros autores ${ }^{68}$.

Este quadro, associado com outras investigações sobre a resistência in vitro do $P$. falciparum em diversas localidades da Região Amazônica Brasileira (Tabela 2), sugere que a resistência à cloroquina tem ampla dispersão 
Couto AA, Calvosa VS, Santos MA, Souza JM. Evolução temporal da resistência in vivo do Plasmodium falciparum às drogas antimaláricas em duas áreas da Amazônia Brasileira com distintas características sócio-econômicas e geográficas. Revista da Sociedade Brasileira de Medicina Tropical 28:357-365, out-dez, 1995.

na região, particularmente, em áreas de garimpagem de ouro ${ }^{2518}$ e colonização ${ }^{37}$, onde o nível de transmissão é elevado.

Por outro lado, as drogas amodiaquina e quinino apresentam um perfil instável, dependendo da área e período de estudos, exibindo um nível flutuante de respostas. Quanto à mefloquina, a sensibilidade nas cepas testadas foi total. No entanto, numa análise anterior Di Santi e col $^{6}$ consideraram como limiar de sensibilidade a concentração de 4 pmol para 16 cepas em 51 analisadas, assim distribuídas: oito de Rondôniia, três do Mato Grosso e Pará, um de Goiás e de um local não identificado. Portanto, atribuíu 31,4\% de cepas resistentes para essa droga na Amazônia Brasileira, dados aqui não confirmados.

O fato da resistência do Plasmodium falciparum ser considerada como um problema limitante do controle da malária, particularmente, em áreas de garimpo e colonização, sugere que a utilização das drogas antimaláricas, empregadas nos diversos esquemas terapêuticos, sofram uma constante avaliação, em programas de monitoração da resistência, no sentido de que seja preservado o poder curativo dos esquemas de tratamento, além de garantir, como reserva terapêutica, para tratamento da malária grave, drogas como mefloquina e outras com características farmacocinéticas semelhantes.

\section{SUMMARY}

We evaluated the temporal progression of in vitro P. falciparum resistance to chloroquine, amodiaquine, quinine and mefloquine in two areas with distinct socioeconomical and geograpbical characteristics: Lourenço, in Amapá state and Paragominas, in Para state. The former region is essentially an "open" gold mining camp, whereas the latter is one currently undergoing a colonization settlement process, in addition to expanding economical activities which mainly include cattle raising and wood exploitation. Our results show high resistance rates to chloroquine in the two study areas: $79.8 \%$ and $68.4 \%$ in Lourenço and Paragominas, respectively. Variations in the response of $\mathrm{P}$. falciparum to both amodiaquine and quinine were recorded throughout the study period. On the other band, no mefloquine P. falciparum resistant strains could be identified, despite the tact we bad noted a decrease in sensitivity to this antimalarial drug througbout the study period.
Key-words: Malaria. P. falciparum. In vitro resistance.

\section{AGRADECIMEN'TOS}

Os autores agradecem à $\mathrm{Dr}^{\mathrm{a}}$ Antoniana Krettli pela leitura crítica e sugestões. À Mineração Novo Astro S/A (Lourenço, AP) pelo suporte logístico. Agradecem também o apoio técnico, nas atividades de campo, aos Srs. Edvaldo Santa Rosa e José Maria Nascimento. Especial agradecimento aos técnicos, supervisores e diretor da Fundação Nacional de Saúde, Coordenadoria Regional do Estado do Amapá pela dedicação e apoio. Agradecem, ainda, a colaboração dos pacientes que sem os quais este estudo não seria possivel.

\section{REFERÊNCIAS BIBLIOGRÁFICAS}

1. Brito R, Pinheiro A. Relatório da Secretaria de Saúde do Território Federal de Guaporé, 1954

2. Couto AA, Calvosa VS, Lima JE, Souza JM. Evolução da resistência in vitro do Plasmodium falciparum a antimaláricos em área de prospecção de ouro no Estado do Amapá, entre 1983 e 1990. Revista da Sociedade Brasileira de Medicina Tropical 26:215-220, 1993.

3. Couto AA, Calvosa Vs, Santos MA, Souza JM. Resistência in vitro de cepas de Plasmodium falciparum isoladas no Sul do Estado do Pará, em diferentes períodos: emergência de casos de multiresistência. Revista da Sociedade Brasileira de Medicina Tropical 26:5-9, 1993.

4. Couto AA, Rosário VE, Walliker D. Análise enzimática de 56 amostras de Plasmodium falciparum da bacia Amazônica (Brasil). Revista Brasileira de Malariologia e Doenças Tropicais 35:11-1993.

5. Couto AA, Santos MA, Oliveira SG. Análise da diversidade do Plasmodium falciparum pela caracterização de clones isolados de amostras da Região Amazônica Brasileira. Cadernos de Saúde Pública 2:241-244, 1986.

6. Di Santi SM, Neves VLFC, Boulos M, Dutra AP, Ramos AMSV, Santos M, Barata LCB. Avaliação da resposta do Plasmodium falciparum à cloroquina, quinino e mefloquina (1). Revista do Instituto de Medicina Tropical de São Paulo 30:147-152, 1988.

7. Knowles G, Davidson WL, Jolley D,Alpers MP. The relationship between the in vitro response of 
Couto AA, Calvosa VS, Santos MA, Souza JM. Evolução temporal da resistência in vivo do Plasmodium falciparum às drogas antimaláricas em duas âreas da Amazônia Brasileira com distintas caracteristicas sócio-econômicas e geográficas. Revista da Sociedade Brasileira de Medicina Tropical 28:357-365, out-dez, 1995.

Plasmodium falciparum to chloroquine, quinine and mefloquine.Transactions of the Royal Society of Tropical Medicine and Hygiene 78:146-150, 1984.

8. Kremsner PG, Zotter GM, Feldmeir H, Graninger W, Kollaristsch M, Wiedermann G, Rocha RM, Wernsdorfer WH. In vitro drug sensitivity of Plasmodium falciparum in Acre, Brazil. Bulletin of the World Health Organization 67: 289-293, 1989.

9. Marques AC. Migration and the dissemination of malaria in Brazil. Memórias do Instituto Oswaldo Cruz 81(supl 2):17-30, 1986.

10. Ministério da Saúde - Superintendência de Campanhas de Saúde Pública - Diretoria Regional do Pará. Relatório técnico da operação impacto contra a malária, 1986.

11. Neiva $A$. Uber die bildung einer chininresistent rase des malariaparasiten. Memórias do Instituto Oswaldo Cruz 2:131-140, 1910.

12. Payne D. Practical aspects of the use of the standard World Health Organization in vitro macro and microtest system for the determination of the sensitivity of Plasmodium falciparum to chloroquine, mefloquine, amodiaquine and quinine. MAP/84.2,01-17, 1984.

13. Payne D. Report on development of the World Health Organization standard microtest kit for chloroquine, mefloquine, amodiaquine and quinine: 1979-1985. MAP/86.1, 01-19, 1986.

14. Rieckmann KH, Sax LJ, Campbel GH, Mrena JE. Drug sensitivity of Plasmodium falciparum. An in vitro microthecnique. The Lancet I: 22-23, 1978.

15. Rosário VE, Couto AA, Santos MA, Oliveira SG. Caracterização de Plasmodium falciparum: Estudos realizados no Brasil. In: Instituto Evandro Chagas; 50 Anos de Contribuição às Ciências Biológicas e à Medicina Tropical (Belém-Pará). Fundação Serviços de Saúde Pública 1: 133-154, 1986.

16. Rosário VE, Couto AA, Santos MA, Souza JM. Caracterização de cepas de Plasmodium falciparum coletadas de pacientes recrudescentes. Revista do Instituto de Medicina Tropical de São Paulo 27: 274-278, 1985.

17. Rosário VE, Couto AA, Vasconcelos SMA, Oliveira SG. Cloning and characterization of Plasmodium falciparum strains. Memórias do Instituto Oswaldo Cruz 81(suppl.II): 143-148, 1986.
18. Santos MAV, Couto AA, Oliveira SG, Rosário VE. Estudos in vitro dos níveis de resistência do Plasmodium falciparum a drogas, de 1983 à 1986. Revista do Instituto de Medicina Tropical de São Paulo 29: 346-353, 1987.

19. Sawyer D. Frontier malaria in the Amazon region of Brazil. Type of malaria situation and some implication for control. Working paper prepared for the meeting of TDR/Fieldmal/SC/Amazon 88:3, 1988.

20. Silva JR. Terçã maligna "cloroquina-resistente" uma séria ameaça ao "Hinterland" brasileiro. Tribuna Médica 4:2-6, 1961.

21. Silva JR. Resistência do P. falciparum à ação da cloroquina. O Hospital 60: 43-58, 1961.

22. Souza JM. Epidemiological distribuition of Plasmodium falciparum drug resistance in Brazil and its relevance to the treatment and control of malaria. Memórias do Instituto Oswaldo Cruz 87(supl III): 343-348, 1992

23. Thaithong S, Beale GH, Chutmongkonkul $M$. Variability in drug susceptibility amongst clones and isolates of Plasmodium falciparum. Transactions of the Royal Society of Tropical Medicine and Hygiene 82:33-36, 1988.

24. Uchoa RS, Souza JM, Pinto AYN, Calvosa VSP, Almeida PMM, Silva SB, Santos MA, Abdon NP, Campos JR, Cereja LN. Casos de malária autóctone na grande Belém em 1993. In: Resumos do XXX Congresso da Sociedade Brasileira de Medicina Tropical, Belém p. 410, 1994.

25. Vasconcelos MA, Rosário VE. Testes de sensibilidade in vitro de amostras de Plasmodium falciparum da bacia Amazônica (Brasil). Revista Brasileira de Malariologia e Doenças Tropicais 25: 21-28, 1983.

26. Webster HK, Thaithong $S$, Pavanand $K$, YongVanttchit K, Pinswasdi C, Boudreau EI. Cloning and characterization of mefloquine-resistant Plasmodium falciparum from Thailand. The American Journal of Tropical Medicine and Hygiene 34:1022-1027, 1985.

27. World Health Organization. Instructions for use of the micro-test kit for the assessment of the response of Plasmodium falciparum to chloroquine and mefloquine in vitro. MAP/82.1, 1-9, 1982.

28. World Health Organization. In vitro micro-test (mark II) for the assessement of the response of 
Couto AA, Calvosa VS, Santos MA, Souza JM. Evolução temporal da resistência in vivo do Plasmodium falciparum às drogas antimaläricas em duas áreas da Amazônia Brasileira com distintas características sócio-econômicas e geográficas. Revista da Sociedade Brasileira de Medicina Tropical 28:357-365, out-dez, 1995.

Plasmodium falciparum to chloroquine, mefloquine, quinine, sulfadoxine-pyrimethamine and amodiaquine. MAP/87.2, 1-21, 1987.

29. Young MD, Moore DV. Chloroquine resistance in Plasmodium falciparum. The American Journal of Tropical Medicine and Hygiene 10:317-320, 1961. 